\title{
Chondroitinase ABC-Mediated Plasticity of Spinal Sensory Function
}

\author{
William B. J. Cafferty, Elizabeth J. Bradbury, Malcolm Lidierth, Martyn Jones, Philip J. Duffy, Sophie Pezet, and \\ Stephen B. McMahon \\ Neurorestoration Group, The Wolfson Centre for Age-Related Diseases, King's College London, London SE1 1UL, United Kingdom
}

Experimental therapeutics designed to enhance recovery from spinal cord injury (SCI) primarily focus on augmenting the growth of damaged axons by elevating their intrinsic growth potential and/or by nullifying the influence of inhibitory proteins present in the mature CNS. However, these strategies may also influence the wiring of intact pathways. The direct contribution of such effects to functional restoration after injury has been mooted, but as yet not been described. Here, we provide evidence to support the hypothesis that reorganization of intact spinal circuitry enhances function after SCI. Adult rats that underwent unilateral cervical spared-root lesion (rhizotomy of C5, C6, C8, and T1, sparing C7) exhibited profound sensory deficits for 4 weeks after injury. Delivery of a focal intraspinal injection of the chondroitin sulfate proteoglycan-degrading enzyme chondroitinase $\mathrm{ABC}(\mathrm{ChABC})$ was sufficient to restore sensory function after lesion. In vivo electrophysiological recordings confirm that behavioral recovery observed in ChABC-treated rats was consequent on reorganization of intact C7 primary afferent terminals and not regeneration of rhizotomized afferents back into the spinal cord within adjacent segments. These data confirm that intact spinal circuits have a profound influence on functional restoration after SCI. Furthermore, comprehensive understanding of these targets may lead to therapeutic interventions that can be spatially tailored to specific circuitry, thereby reducing unwanted maladaptive axon growth of distal pathways.

Key words: chondroitin sulfate; plasticity; spinal cord injury; traumatic injury; dorsal rhizotomy; proteoglycan

\section{Introduction}

Axonal architecture within the adult CNS is the product of coordinated neurite outgrowth, axonal path finding during development and a period of activity-dependent refinement during the early postnatal epoch (Tessier-Lavigne, 2002). Mature circuits are relatively stable after the closure of defined critical periods (Hensch, 2004), and accumulating evidence suggests (Pizzorusso et al., 2002, 2006; McGee et al., 2005) that adult CNS neurons are restricted from large-scale arbor reorganization by two broad classes of inhibitor proteins present throughout the mature neuroaxis, namely, myelin-associated inhibitors (MAIs) (Liu et al., 2006) and chondroitin sulfate proteoglycans (CSPGs) (Busch and Silver, 2007). Although these proteins efficiently restrict potentially maladaptive axonal sprouting in the intact adult CNS (Maier and Schwab, 2006), their continued adult expression also prevents axotomized neurons regenerating and recapitulating

\footnotetext{
Received Aug. 14, 2008; accepted Sept. 9, 2008.

This work was supported by The Wellcome Trust. W.B.J.C. is supported by a National Institutes of Health K99/R00 award.

Correspondence should be addressed to Stephen B. McMahon, Neurorestoration Group, The Wolfson Centre for Age-Related Diseases, King's College London, The Wolfson Wing, Hodgkin Building, Guy's Campus, London Bridge, London SE1 1UL, UK. E-mail: stephen.mcmahon@kcl.ac.uk.

W. B. J. Cafferty's present address: Program in Cellular Neuroscience, Neurodegeneration, and Repair, Yale University School of Medicine, 295 Congress Avenue, BCMM 433, New Haven, CT 06510.

S. Pezet's present address: Laboratoire de Neurobiology, Centre National de la Recherche Scientifique, Unité Mixte de Recherche 7637, Ecole Supérieure de Physique et de Chimie Industrielles, 10 rue Vauquelin, 75005 Paris, France. DOl:10.1523/JNEUROSCI.3877-08.2008

Copyright $\odot 2008$ Society for Neuroscience $\quad$ 0270-6474/08/2811998-12\$15.00/0
}

function after brain or spinal cord injury (Bradbury and McMahon, 2006).

Restoration of functionally meaningful behaviors after spinal cord injury (SCI) requires communication between regenerating axons and their physiological targets, which can be separated by many centimeters. Currently, most experimental SCI studies in rodents that use antiinhibitory strategies to enhance growth of damaged descending spinal tracts have shown the presence of regenerating axons a maximum of millimeters caudal to a lesion (Bradbury and McMahon, 2006). Therefore, most axons stop growing some distance away from regions that could mediate return of function, for example, corticospinal axon growth into the lumbar spinal cord after thoracic cord damage. Despite the lack of long-distance axonal regeneration observed, these strategies have succeeded in improving motor function after SCI (Bradbury and McMahon, 2006; Maier and Schwab, 2006; Busch and Silver, 2007). This apparent dichotomy points to a complementary action of these therapies on circuits that are not damaged as well as promoting regeneration of damaged tracts. Consequently, local sprouting and plasticity of intact spinal circuits driven by mitigation of the effects of inhibitory proteins by anti-MAI and antiCSPG therapies is thought to be at least partially responsible for the observed recovery of function in these studies (Bareyre et al., 2004; Kim et al., 2004; Cafferty and Strittmatter, 2006).

The presence of spared tissue bridges after SCI is a prerequisite for the ability of intact circuits caudal to a lesion to be accessed from brain circuits and to allow some voluntary control of them. Most human SCIs are incomplete and there is usually some lim- 
ited functional recovery enhanced by intensive rehabilitative therapy (Maier and Schwab, 2006; Baptiste and Fehlings, 2007). Reorganization of intact circuitry is presumably responsible. Selective peripheral nerve and CNS lesions in animal studies have revealed that axonal sprouting and alterations in central connectivity does occur and can have profound functional impact (Wilson and Kitchener, 1996; Bareyre et al., 2004).

Therefore, understanding the mechanism by which intact circuits can sprout into, and restore function to, structures that have lost synaptic input would greatly enhance the potential for functional restoration after SCI. Recent studies have shown that ocular dominance plasticity can be restored after the closure of the critical period in rodents after treatment with an enzyme that digests CSPGs, chondroitinase ABC (ChABC) (Pizzorusso et al., 2002, 2006). These studies suggest that the presence of CSPGs in the extracellular matrix of the visual cortex restricts experiencedependent synaptic plasticity (Fox and Caterson, 2002; Berardi et al., 2004). CSPGs are synthesized by both neurons and glial cells throughout spinal gray and white matter (Tang et al., 2003) and are the major inhibitory constituent of the glial scar that characterizes CNS lesions (Davies et al., 1999; Asher et al., 2000, 2002; Jones et al., 2002, 2003; Tang et al., 2003). In vivo delivery of ChABC has been shown to encourage functional axonal regeneration (Zuo et al., 1998; Yick et al., 2000; Moon et al., 2001; Bradbury et al., 2002; Caggiano et al., 2005), and sprouting (Steinmetz et al., 2005; Barritt et al., 2006; Massey et al., 2006, 2008) after SCI.

Here, we have designed experiments to assess the ability of ChABC to functionally reorganize spinal connectivity after partial deafferentation of the spinal cord. We demonstrate for the first time that digestion of CSPGs leads to a highly significant reorganization of spinal circuitry and a significant recovery of function mediated by undamaged afferent inputs. Our results demonstrate an important mechanism that limits functional reorganization in the adult CNS and suggest opportunities to promote recovery of function after CNS injury.

\section{Materials and Methods}

\section{Surgical procedures}

Spared-root lesions were completed on 101 (sham, 18; lesion, 83) adult male Wistar rats (Charles River). Under inhalation anesthesia (2\% isoflurane in $\mathrm{O}_{2}$ ), animals underwent a hemilaminectomy from C5 to T1. Dorsal roots C5, C6, C8, and T1 on the left side were crushed three times for $10 \mathrm{~s}$, and C7 was spared. Concomitantly, animals received a unilateral microinfusion of either $1 \mu \mathrm{l}$ of saline $(n=41)$ or $1 \mu \mathrm{l}$ of protease-free ChABC $(0.01 \mathrm{U})$ (Seikagaku Corporation) $(n=42)$ into the left dorsal horn at C7. The wound was closed in layers, and the animals were returned to their home cages where they received food and water ad libitum. Five days before killing, animals were anesthetized with $2 \%$ isoflurane and received bilateral injections of $5 \mu \mathrm{l}$ of $\beta$-subunit cholera toxin (CTB) (0.1\%; List Laboratories) into the C7 dermatome. Experimental procedures conformed with, and were licensed under, United Kingdom legislation (Animals Scientific Procedures Act, 1986).

\section{Immunohistochemical analysis}

Animals were deeply anesthetized with pentobarbitone $(80 \mathrm{mg} / \mathrm{kg}$, i.p.) and transcardially perfused with $100 \mathrm{ml}$ of $0.1 \mathrm{M}$ phosphate buffer followed by $4 \%$ paraformaldehyde (PFA). Spinal cords were dissected free and postfixed overnight at $4{ }^{\circ} \mathrm{C}$. Tissue was then embedded in $10 \%$ gelatin (Sigma-Aldrich). Gelatin blocks were fixed for $18 \mathrm{~h}$ in $4 \% \mathrm{PFA}$, and then stored in $0.01 \mathrm{~m}$ PBS until sectioning. Spinal cords were sectioned on a vibrating microtome (Leica) in the coronal or sagittal plane at $30 \mu \mathrm{m}$. Standard direct immunohistochemical procedures were performed to detect the following antigens: chondroitin-4-sulfate (C4S) (ICN; 1:1K), GFAP (Dako; 1:10K), neurocan (1F6; Developmental Studies Hybridoma Bank; 1:1K), phosphacan (3F8; Developmental Studies Hybridoma Bank; 1:1K), phospho-p44/42 MAPK (mitogen-activated protein kinase)
(Cell Signaling Technology; 1:400) and neuronal-specific nuclear protein (NeuN) (A60 clone; Millipore; 1:1K), CTB (List Laboratories; 1:5000), and calcitonin gene-related peptide (CGRP) (Sigma-Aldrich; 1:10K). Anti-mouse, -rabbit, and -goat secondary antibodies conjugated to Alexa Flour 488 and 568 were used to detect signal. Some sections were stained with DAPI (4',6'-diamidino-2-phenylindole) (Invitrogen; 1:10K). Sections were mounted on Superfrost Plus slides and coverslipped with Vectashield (Vector Laboratories). Sections were viewed with a Zeiss Imager microscope (Zeiss).

\section{Immunoblotting}

Spinal segments C5-T1 from lesioned and treated animals were freshly dissected, and individual hemisegments (see Fig. $1 \mathrm{~A}$ ) were immediately homogenized in lysis buffer $(20 \mathrm{~mm}$ Tris, $\mathrm{pH} 8,137 \mathrm{~mm} \mathrm{NaCl}, 10 \%$ glycerol, $1 \%$ Nonidet P-40, 2 mM EDTA, 1 mM PMSF, $10 \mu \mathrm{g} / \mathrm{ml}$ antipapain, $10 \mu \mathrm{g} / \mathrm{ml}$ leupeptin, $1 \mathrm{~mm}$ sodium vanadate, $5 \mathrm{~mm} \mathrm{NaF}, 1 \mu \mathrm{g} / \mathrm{ml}$ pepstatin; Sigma-Aldrich) at $4^{\circ} \mathrm{C}$ with an Ultra-Turax. Lysates were left for $2 \mathrm{~h}$ to rotate at $4^{\circ} \mathrm{C}$ and were then centrifuged at $12,000 \times g$ for $20 \mathrm{~min}$ at $4^{\circ} \mathrm{C}$. Supernatants were collected and total protein concentration was determined using a BCA kit (Pierce). Some lysates were incubated with $0.001 \mathrm{U}$ of $\mathrm{ChABC}\left(2 \mathrm{~h}\right.$ at $\left.37^{\circ} \mathrm{C}\right)$ to digest all sulfated CSPGs. Lysates were then boiled in loading buffer, and $15 \mu \mathrm{g}$ of protein was electrophoresed on $5 \%$ acrylamide gel. After transfer, PVDF (polyvinylidene difluoride) membranes were probed overnight for chondroitin-4-sulfate (ICN; $1: 1 \mathrm{~K})$. Visualization was performed using ECL Plus solution and exposure onto X films (GE Healthcare). Films were scanned and analyzed in NIH Image J, version $1.37 \mathrm{v}$.

\section{Behavioral assessment}

Animals underwent two behavioral tests after lesion.

Spontaneous vertical exploration [adapted from the study by Schallert et al. (2000)]. Rats were placed in a clear Perspex cylinder for $5 \mathrm{~min}$. A mirror was placed at an angle behind the cylinder so that the forepaws could be viewed at all times. The testing session was videotaped (Sony digital video camera recorder; DCR-TRV60E), and an experimenter blinded to the treatment group scored forepaw use at a later date. The animals required no pretraining but were placed in the cylinder twice before surgery for habituation and to obtain baseline scores. The use of ipsilateral (ipsi) and contralateral (contra) paws was observed, and the number of initiating (vertical) and exploratory (lateral) movements made by each paw was tallied. Animals were assessed on days 2, 7, 14, and 21 after lesion. Data were analyzed using a multivariate ANOVA with repeated measures.

Tape removal. Rats had a square of adhesive tape placed on the palmar surface of their lesioned forepaws, and the time taken to identify the tape (the "sense" component) and the time taken for the rats to remove the tape after sensing it (the "motor" component) were recorded once before lesion and on days 2, 7, 14, and 21 after lesion. Data were analyzed using a multivariate ANOVA with repeated measures.

\section{In vivo electrophysiological recordings}

Animal preparation. Experiments were performed on adult male Wistar rats $(n=35)$ anesthetized with $1.25 \mathrm{~g} / \mathrm{kg}$ urethane (Sigma-Aldrich). At the end of each experiment, animals were killed by an anesthetic overdose. The trachea and jugular vein were cannulated, and animals were mounted in a stereotaxic frame providing support via ear bars and pelvic clamps. Rectal temperature was monitored and used to regulate a homoeothermic blanket. The electrocardiogram was recorded via percutaneous electrodes in the right and left forelimbs. A bilateral laminectomy was made to expose the spinal cord from C3 to T3. The spinal cord was further stabilized with spinous clamps on the $\mathrm{C} 2$ and $\mathrm{T} 2$ dorsal spinous processes. The surrounding retracted musculature formed a pool, which was filled with warm paraffin to protect the exposed spinal cord. The dura mater was gently removed and a small square of insulation material was placed between the $\mathrm{C} 7$ dorsal roots and the spinal cord to minimize passive current spread from subsequent electrical stimulation of the root. For recording, gallamine triethiodide $(20 \mathrm{mg}$, i.v.) was administered to achieve neuromuscular blockade and the rat was artificially ventilated. Expired $\mathrm{CO}_{2}$ was monitored, and ventilation volume adjusted, to maintain an end-tidal $\mathrm{CO}_{2}$ concentration of $3-4 \%$. 
Cord dorsum potentials. Threshold stimulus for recording cord dorsum potentials (CDPs) was determined by stimulating the intact C7 dorsal root with a tungsten microelectrode and recording activity at the C7 dorsal columns. Increasing the stimulus magnitude from 0.1 to 100 $\mu \mathrm{A}(200 \mu$ s pulse at $0.5 \mathrm{~Hz})$, revealed a threshold of $\sim 10 \mu \mathrm{A}$. Thereafter, a $5 \times$ threshold ( $50 \mu \mathrm{A} ; 200 \mu$ s pulse) at $0.5 \mathrm{~Hz}$ protocol was used to elicit CDPs in treated animals along the dorsal columns from C5 to T1, after $\mathrm{C} 7$ root stimulation. All recordings were digitized and averaged using a PC and interface card (CED Micro1401 MkII) with Signal or Spike2 for Windows software (Cambridge Electronic Design). CDPs (average of 30 sweeps) were compared between treatment groups using ANOVA.

Field potential recording. Focal field potentials elicited by C7 dorsal root stimulation (using the same parameters as above) were recorded with a tungsten microelectrode in a series of three tracts made 400,600 , and $800 \mu \mathrm{m}$ lateral from the midline and extending from the cord surface down to a depth of $1.2 \mathrm{~mm}$ separated by $100 \mu \mathrm{m}$ steps. The $\mathrm{N}$-waves from field potential recordings (average of 30 sweeps) were compared between treatment groups using ANOVA.

Single-unit recordings. Single units were identified via natural stimulation of the forepaw. A tungsten microelectrode was driven the $\mathrm{C} 7 \mathrm{dorsal}$ horn while gently tapping or brushing the digits, arm, and shoulder region of the rat. Amplified and filtered signals were displayed on an oscilloscope and fed into an audio amplifier (Neurolog). Single units were initially identified as having uniform spike amplitude and being clearly distinguishable ( $>3: 1$, signal-to-noise) from background neuronal noise. Once isolated units were characterized with respect to depth, response to natural stimuli, spontaneous activity, and receptive field size. The receptive field was mapped by blunt stimulation of the forelimb, and its extent was recorded on a forelimb map. The responsiveness of the cell was also characterized by stimulating the center of its receptive field with pin electrodes and recording poststimulus time histograms (PSTHs).

Noxious stimulus [adapted from the study by Ji et al. (1999)]. Under sodium pentobarbital anesthesia (60 mg per $\mathrm{kg}$, i.p.), 8-methyl- $n$ vanillyl-6-noneamide (capsaicin) (Sigma-Aldrich; $3 \mathrm{mg}$ per ml dissolved in $10 \%$ Tween 80 ) was injected into the C7 dermatome (25 $\mu \mathrm{l} ; 75 \mu \mathrm{g})$. Animals were left to recover for $5 \mathrm{~min}$, and then perfused with $0.1 \mathrm{M}$ phosphate buffer followed by $4 \%$ paraformaldehyde with $1.5 \%$ picric acid, and C5-T1 spinal cord was removed and postfixed. Tissue was then prepared for immunohistochemistry.

\section{Results \\ Chondroitinase $\mathrm{ABC}$ digests intrinsic spinal chondroitin sulfate proteoglycans}

We used focal microinjections of the bacterial enzyme ChABC to digest GAG side chains from endogenous CSPGs in the adult rat spinal cord (Fig. $1 A$ ). A single in vivo injection of $1 \mu \mathrm{l}$ of $0.01 \mathrm{U}$ ChABC or vehicle (saline) was made in the left C7 dorsal horn, and tissue was harvested $14 \mathrm{~d}$ later. Individual spinal hemisegments (ipsi and contra) (Fig. $1 A$ ) were analyzed by Western blotting for the presence of digested CSPGs using the antibody C4S, which detects all digested 4-sulfated CSPGs. Naive C7 hemisegments treated in vitro with $0.01 \mathrm{U}$ of $\mathrm{ChABC}$ revealed that C4Spositive bands resolve at a range of molecular weights corresponding to the array of 4-sulfated CSPGs that are present in the adult spinal cord (Fig. $1 B$, labeled $\mathrm{C} 7, \mathrm{~N}+$ ). Vehicle injection into the $\mathrm{C} 7$ segment or vehicle treatment of naive spinal cord treated in vitro with $\mathrm{PBS}$ did not elicit significant CSPG hydrolysis (Fig. $1 B$, labeled $\mathrm{C} 7, \mathrm{~N}-$ ). Spinal segments surrounding the ChABC injection site showed robust digestion of intrinsic CSPGs. Densitometric analysis allowed quantification of the percentage of ChABCmediated digestion of 4-sulfated CSPGs observed in vivo compared with in vitro digestion (i.e., $100 \%$ digestion). Figure $1 C$ summarizes the extent of digestion observed for tissue harvested $14 \mathrm{~d}$ after in vivo ChABC injection, and statistically significant $\left({ }^{*} p<0.005\right.$, ANOVA) hydrolysis of 4-sulfated CSPGs was observed in contralateral segments $\mathrm{C} 7$ and $\mathrm{C} 8$ and ipsilateral segments C7, C8, and T1. Evidence of ChABC-mediated digestion of intrinsic CSPGs was apparent up to $28 \mathrm{~d}$ after injection (data not shown).

The efficacy of a single in vivo ChABC injection to digest endogenous CSPGs was also confirmed by immunohistochemical analysis. C4S antibody was used to stain either sagittal or transverse sections of spinal cord (Fig. $1 D, E$ ) through the injection site. In vivo $\mathrm{ChABC}$ injection resulted in widespread hydrolysis of GAG chains throughout the gray matter in the treated spinal segments, and to a lesser extent in adjoining segments both ipsilaterally and contralaterally. Vehicle-treated animals failed to reveal C4S immunoreactivity (data not shown).

The central hypothesis behind these experiments is that digestion of CSPGs in the spinal cord will facilitate changes in synaptic connectivity. We chose to examine this under conditions in which such changes are more likely to be revealed, in a classic "spared-root" preparation. Therefore, at the time of ChABC treatment, we crushed two rostral and two caudal dorsal roots ipsilateral to the injected spinal segment (i.e., C5, C6, and C8, and $\mathrm{T} 1$ were crushed, sparing the $\mathrm{C} 7$ root (Fig. $1 A$ ). Immunohistochemical analysis revealed that this procedure did not itself produce any digestion of CSPGs within intact (Fig. $1 F$ ) or rhizotomized (Fig. 1I) spinal segments, as seen with the C4S antibody, nor did it alter the expression of two specific CSPGs, neurocan (Fig. $1 G, J$ ) and phosphacan (Fig. $1 H, K$ ), which are both highly expressed in spinal gray matter before and after rhizotomy.

\section{Chondroitinase $\mathrm{ABC}$ normalizes deafferentation-induced compromise of sensory function}

The spared-root preparation used here disconnects most of the sensory innervation from the forepaw to the spinal cord, and leads to behavioral deficits in the use of the affected forepaw. We undertook two behavioral tests to assess the ability of ChABC to restore sensory function to lesioned animals. First, we measured spontaneous vertical exploration. Animals were placed in a transparent cylindrical chamber (Fig. $2 A$ ), and the use of forepaws to initiate vertical and lateral movements during spontaneous vertical exploration was scored (Fig. 2A). In intact animals (sham lesioned, $n=8$ ), both forepaws were used equally, and most exploratory rearing movements used both paws simultaneously. Sham lesion did not significantly affect this behavior. Animals with a spared-root injury (unilateral rhizotomies of C5-T1 but sparing $\mathrm{C} 7, n=7$ ) showed a large reduction of $>50 \%$ on the usage of the ipsilateral paw at day 3 after lesion, and then a more modest but still significantly reduced use of the injured paw up to $21 \mathrm{~d}$ after injury $\left({ }^{*} p<0.01\right.$, multivariate repeated-measures ANOVA). In contrast, spared-root-lesioned animals that received intraspinal ChABC $(n=8)$ showed significant recovery of forelimb symmetry at all times examined compared with sparedroot-lesioned animals that had received saline (Fig. 2 A). ChABCtreated animals showed impairment on day 3 after surgery (but only about one-half as much as the saline-treated animals) and at all later time points appeared indistinguishable from the shamlesioned animals.

A tape removal task measured both sensory and motor function of the injured forelimb. Sham-lesioned animals initially took $\sim 5-10$ s to sense the tape (Fig. $2 \mathrm{~B}$ ) on their paws and an additional 5-10 s to remove the tape (Fig. 2C). Time taken for animals to sense and remove the tape decreased over the testing period, presumably because of familiarity with the task. Animals that underwent spared-root injury and saline treatment took significantly longer to notice the tape on their forelimbs (Fig. $2 B$ ); this lesion-induced deficit was most pronounced at $3 \mathrm{~d}$ and slowly improved thereafter, reaching levels seen in the sham animals by 
A

Spared root preparation
B

Spinal level $\quad \mathrm{C} 7 \mathrm{C}_{5} \mathrm{C} 5 \mathrm{C}_{6} \mathrm{C} 6 \mathrm{C}_{7} \mathrm{C} 7 \mathrm{C} 7 \mathrm{C} 8 \mathrm{C} 8 \mathrm{~T} 1 \mathrm{~T} 1$ Experimental side $\mathrm{N}$ ip con ip con ip con $\mathrm{N}$ ip con ip con ChABC in vivo $-+++++++_{+}+++$ ChABC in vitro . . . . . . + . .
C

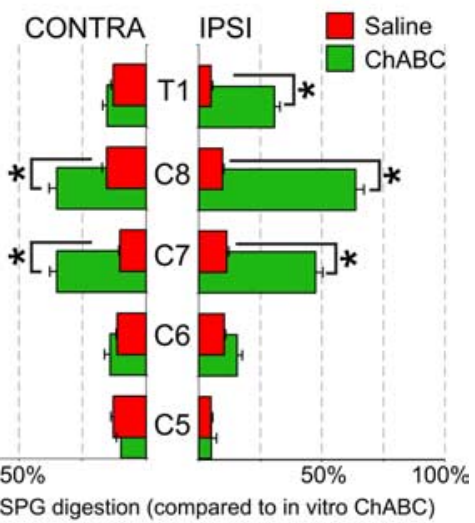

$\%$ CSPG digestion (compared to in vitro ChABC)
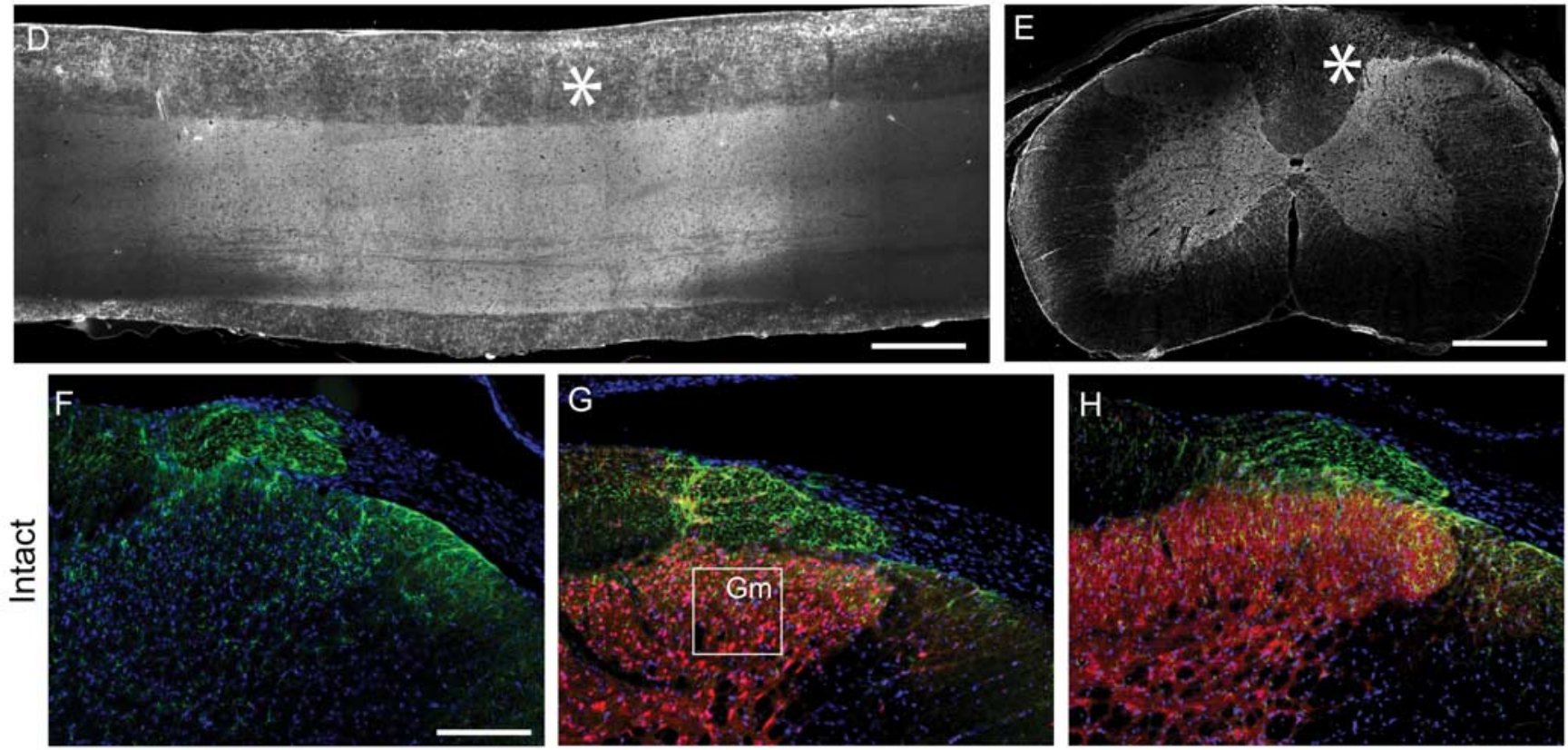

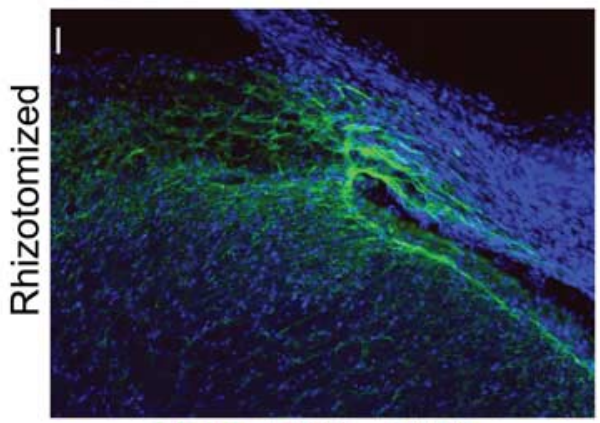

GFAP C4S DAPI

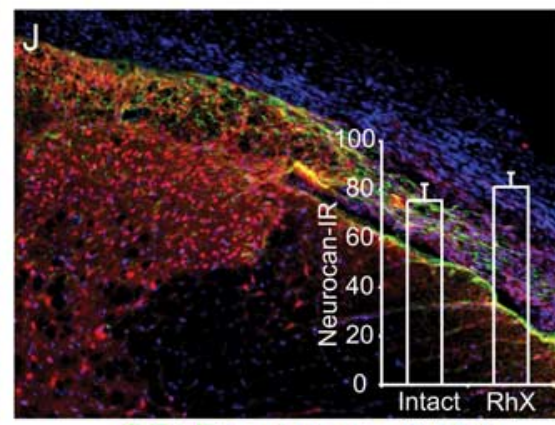

GFAP neurocan DAPI

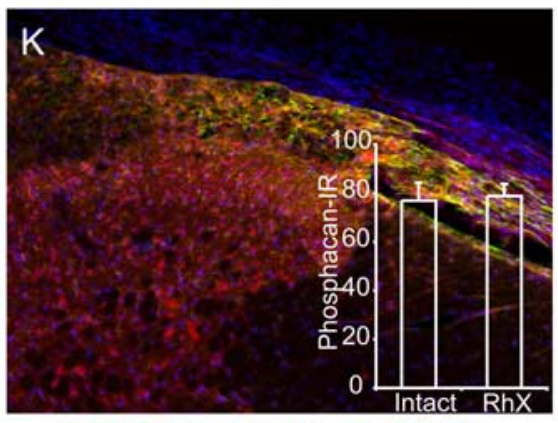

GFAP phosphacan DAPI

Figure 1. In vivo digestion of CSPGs with chondroitinase $A B C$. $A$, Schematic diagram illustrating the site of a single microinjection of ChABC or saline (shaded area in C7 spinal segment) and relative location of rhizotomized dorsal roots $(\mathrm{C} 5, \mathrm{C} 6, \mathrm{C} 8$, and T1). Ipsilateral and contralateral spinal cord segments from animals microinjected with saline or $\mathrm{Ch} A B C$ were harvested for protein analysis $14 \mathrm{~d}$ after lesion and treatment. $\boldsymbol{B}$, Immunoblot analysis of spinal cord lysates probed with anti-chondroitin-4-sulfate antibody (C4S) revealed digestion of all endogenous 4-sulfated CSPGs $14 \mathrm{~d}$ after ChABC delivery [N indicates naive spinal cord lysate from a control animal incubated in vitro in the presence $(+)$ or absence $(-)$ of $C h A B C]$. C, Analysis of lysates from experimental animals treated with saline (red bars) or ChABC (green bars) revealed that ChABC injected unilaterally at C7 diffused bilaterally and significantly $\left({ }^{*} p<0.005\right.$, ANOVA) digested 4-sulfated CSPGs from C7 to T1. Photomicrograph of a sagittal $(\boldsymbol{D})$ and a transverse $(\boldsymbol{E})$ section of spinal cord $14 \mathrm{~d}$ after ChABC microinjection (asterisk, injection site) immunostained with C4S. Digestion of intrinsic 4-sulfated CSPGs can be seen spreading both rostrocaudally and mediolaterally from the injection site. Scale bars: $\boldsymbol{D}, \boldsymbol{E}, 500 \mu \mathrm{m}$. Photomicrographs of transverse sections from $\boldsymbol{C 7}$ [intact segment $(\boldsymbol{F}-\boldsymbol{H})$ ] and C8 [rhizotomized segment $(\boldsymbol{I}-\boldsymbol{K})$ ] after spared-root lesion. Spared-root lesion does not digest intrinsic CSPGs within intact $(\boldsymbol{F})$ and rhizotomized $(\boldsymbol{I})$ segments, indicated by the absence of C4S immunoreactivity. Neurocan (red) is highly expressed in spinal gray matter $(\mathrm{Gm})$ within intact segment $\mathrm{C}(\boldsymbol{G})$ and does not increase after rhizotomy (Rhx) $(\boldsymbol{J})$. Phosphacan $(\mathrm{red})$ is also highly enriched in spinal gray matter within intact $\mathbf{C}(\boldsymbol{H})$ and is unaltered after rhizotomy $(\boldsymbol{K})$. Scale bar, $200 \mu \mathrm{m}$. Quantification of $(S P G$ expression within dorsal horn gray matter $(\boldsymbol{G}$, boxed area) confirms that both neurocan and phosphacan levels are constitutively high in spinal gray matter and remain unchanged after RhX (bar graphs in $\boldsymbol{J}$ and $\boldsymbol{K}$, respectively). Error bars indicate SEM. 

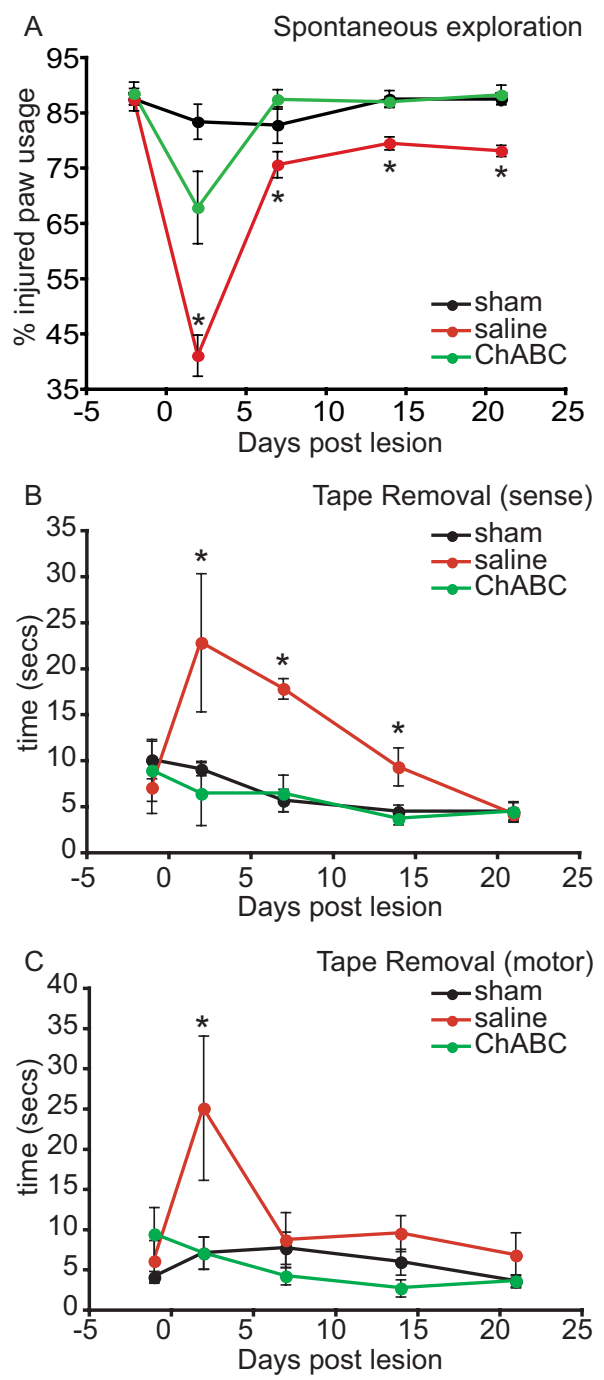

Figure 2. Chondroitinase $A B C$-mediated restitution of sensory function after spared-root lesion. A, During spontaneous exploration of a Plexiglas cylinder (assessed over $21 \mathrm{~d}$ ), intact animals use both forelimbs simultaneously to vertically explore the cylinder $\sim 50-60 \%$ of the time; the reminder of the time individual paws are used. The experimental forelimb (left) score was $\sim 85 \%$ (injured limb plus both limbs) presurgery. Spared-root lesion and saline treatment resulted in a significant deficit in the ability of the animals to explore laterally with their injured forelimb throughout the experimental period. In contrast, spared-root lesion animals treated with ChABC illustrated a deficit at only at $2 \mathrm{~d}$ after lesion, but recovered and were indistinguishable from sham-lesioned control animals within $7 \mathrm{~d}\left({ }^{*} p<0.01\right.$, multivariate ANOVA). B, C, In an adhesive tape removal task, spared-root-lesioned animals treated with saline displayed a significant deficit in sense scores up to $14 \mathrm{~d}$ after injury and motor scores at $2 \mathrm{~d}$ after lesion, compared with sham-lesioned control animals. In contrast, spared-root-lesioned animals treated with $C h A B C$ failed to show a deficit at any time point after injury compared with sham animals $\left({ }^{*} p<0.01\right.$, multivariate ANOVA). Error bars indicate SEM.

day 21 . The ability of these lesioned animals to remove the tape was only impaired on day 3 after lesion and was normal thereafter (Fig. $2 C)\left({ }^{*} p<0.01\right.$, multivariate repeated-measures ANOVA). Spared-root-lesioned animals treated with ChABC did not show a deficit in their ability to sense the presence of the tape (Fig. $2 B$ ) or the ability to remove the tape (Fig. $2 C$ ) on any of the postinjury testing days. Thus, intraspinal delivery of ChABC significantly improved behavioral performance in both functional paradigms.

\section{Chondroitinase $\mathrm{ABC}$ promotes anatomical reorganization of intact fibers}

Parsimoniously, two mechanisms could mediate the restoration of sensory function in animals treated with ChABC. We visual- ized lesioned fibers using the intrinsic marker CGRP and intact fibers using the extrinsic marker $\mathrm{CTB}$ via transganglionic tracing of the $\mathrm{C} 7$ dermatome.

Regenerating CGRP-immunoreactive (IR) axons were consistently observed to stop growing on reaching the damaged dorsal root entry zone (DREZ) in both vehicle- and ChABC-treated animals (supplemental Fig. 1, available at www.jneurosci.org as supplemental material). Because of the extensive segmental overlap of primary afferent terminals innervating the spinal cord through the brachial plexus, intrinsic markers such as CGRP cannot differentiate between intact $\mathrm{C} 7$ fibers and intact fibers entering the spinal cord through C4 and T2. As expected, assessment of primary afferent terminal density with CGRP failed to reveal a difference between treatment groups (supplemental Fig. 1, available at www.jneurosci.org as supplemental material). By injecting CTB bilaterally into the C7 dermatomes, we were able to visualize the rostrocaudal extent of the intact $\mathrm{C} 7$ fibers innervation in both saline- and ChABC-treated animals. CTB-IR terminals were observed in lamina III-VI on the contralateral (Fig. $3 A-D$ ) and ipsilateral (Fig. $3 F-I$ ) side of saline-treated animals and on the contra (Fig. $3 K-N$ ) and ipsi (Fig. $3 P-S$ ) side of ChABC-treated animals. By measuring the mediolateral limits of CTB immunoreactivity through the lamina III-IV border, we were unable to detect a difference in CTB terminal distribution contralateral to the lesion in either saline- or ChABC-treated animals (Fig. 3, compare E, O). The extent of CTB-IR terminals was significantly reduced ipsilateral to the lesion in saline-treated animals (Fig. $3 F-J, U)\left({ }^{*} p<0.01\right.$, ANOVA). However, CTB terminal distribution was significantly greater in ChABC-treated animals ipsilateral to the lesion compared with saline-treated animals (Fig. $3 P-U)\left({ }^{*} p<0.01\right.$, ANOVA). That is, ChABC treatment allows anatomical sprouting of CTB-labeled fibers under these conditions.

\section{Chondroitinase $\mathrm{ABC}$ restores postsynaptic activity after spared-root injury}

We further tested the idea that the recovery of behavioral function induced by intraspinal ChABC after spared-root lesion resulted from altered spinal connectivity of the intact sensory fibers supplying the forepaw (i.e., those projecting through the $\mathrm{C} 7 \mathrm{dor}$ sal root), using in vivo electrophysiology. Animals that underwent spared-root injury were prepared for recording $7 \mathrm{~d}(n=5)$, $14 \mathrm{~d}(n=5)$, and $28 \mathrm{~d}(n=5)$ after spared-root lesion and ChABC $(n=15)$ or saline $(n=15)$ treatment. Animals underwent systematic recording of CDPs in response to electrical stimulation of the $\mathrm{C} 7$ dorsal root (Fig. $4 A, B$ ). Dorsal root stimulation resulted in reproducible CDPs. The waveform in Figure $4 A$ shows a stimulus artifact followed by a slow $\mathrm{N}$-wave, the latter being an expression of underlying postsynaptic activity. The threshold stimulus was $\sim 10 \mu \mathrm{A}$ (using a $200 \mu$ s pulse), and increasing the stimulus in $10 \mu \mathrm{A}$ steps revealed a maximal CDP of approximately $-2.0 \mathrm{mV}$ with $100 \mu \mathrm{A}$ stimulation in intact animals (Fig. $4 B$, black circles). Acute rhizotomy of the C7 dorsal root abolished all CDPs (Fig. $4 B$, open circles). Spared-root lesion and saline microinjection led to significantly reduced CDPs (Fig. $4 B$, red circles) $\left({ }^{*} p<0.05\right.$, ANOVA). Fourteen days after lesion, CDPs from these animals reached a maximum of approximately $-0.5 \mathrm{mV}$ suggestive of a lesion-induced reduction in responsiveness of second-order neurons. Animals that received ChABC microinjection with spared-root lesion did not show a significant lesion-induced reduction in CDP, with CDPs that reached $-2.0 \mathrm{mV}$ at $100 \mu \mathrm{A}$ stimulation, significantly higher compared with saline-treated animals ( $p<0.05$, ANOVA), but 

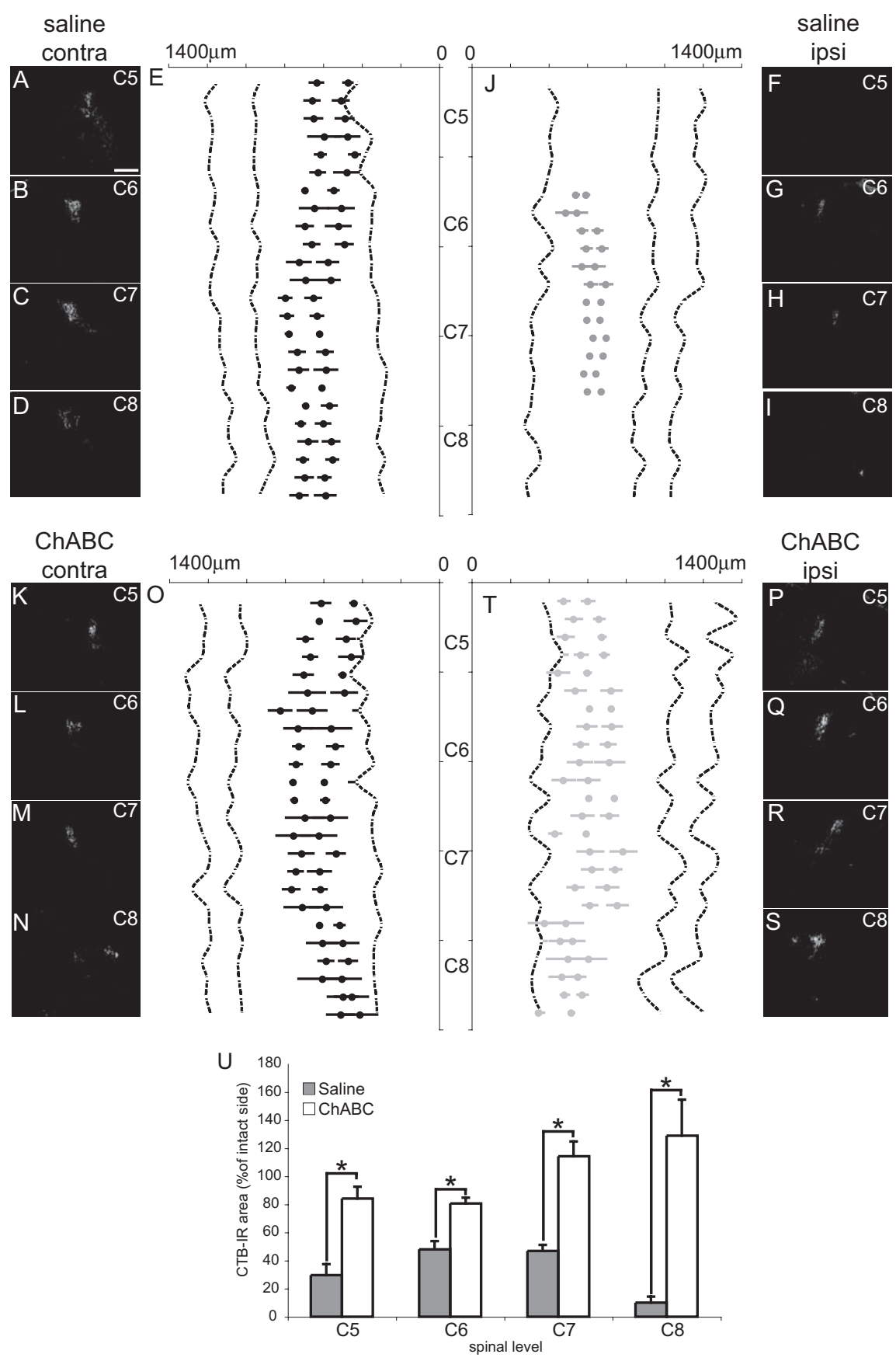

Figure 3. Chondroitinase $A B C$ enhances anatomical reorganization of intact CTB-IR fibers. Photomicrographs illustrate CTB-IR primary afferent terminals in contralateral $(\boldsymbol{A}-\boldsymbol{D})$ and ipsilateral $(\boldsymbol{F}-\boldsymbol{I})$ spinal cord in saline-treated animals and contralateral $(\boldsymbol{K}-\boldsymbol{N})$ and ipsilateral $(\boldsymbol{P}-\boldsymbol{S})$ spinal cord in ChABC-treated animals after spared-root lesion. Horizontal dorsal horn mappings $(\boldsymbol{E}, \boldsymbol{J}$, $\mathbf{O}, \boldsymbol{T})$ illustrate the mediolateral distribution of (TB-IR terminals at the level of the lamina III-IV border after peripheral C7 dermatome (TB injections in spared-root-lesioned animals after vehicle $(\boldsymbol{E}, \boldsymbol{J})$ or $\mathrm{ChABC}$ treatment $(\boldsymbol{O}, \boldsymbol{T})$. In each graph, the midline is set at $0 \mu \mathrm{m}$, and the lateral extent of the dorsal columns is marked by the first dashed line; the gray matter extends between the first and second dashed lines, and the dorsolateral columns extend between second and third dashed lines. On this map, the area occupied by CTB-IR terminals is marked by the horizontal lines at different rostrocaudal levels. The rostrocaudal extent of CTB-IR terminal distribution is significantly reduced at all spinal levels on the ipsilateral side in saline treatment compared with $C h A B C$ treatment $(\boldsymbol{U})\left({ }^{*} p<0.001\right.$, one-way ANOVA), as shown by the histogram below, in which data are expressed as average \pm SEM mediolateral occupation of CTB-IR terminals.

statistically indistinguishable from control intact animals (Fig. $4 B$, green circles).

CDPs were also systematically recorded from spinal levels C5 through T1 along the ipsilateral dorsal columns using $50 \mu \mathrm{A} \times$ $200 \mu$ s stimulation of the intact C7 dorsal root. As expected, the
$\mathrm{N}$-wave in intact animals was largest in the spinal C7 segment and decreased in adjacent segments (Fig. 4C). Animals that received spared-root lesion and saline treatment displayed significantly reduced CDPs at all segments assessed compared with intact animals (Fig. $4 C)\left({ }^{*} p<0.05\right.$, ANOVA), whereas animals that had undergone spared-root lesion and $\mathrm{ChABC}$ treatment displayed CDPs at all spinal segments equivalent to (and statistically indistinguishable from) that seen in intact animals (Fig. 4C). The data in Figure 4 relate to experiments undertaken $14 \mathrm{~d}$ postoperatively, but similar results were seen at 7 and $28 \mathrm{~d}$ (data not shown).

We next made focal field potential recordings within the intact $\mathrm{C} 7$ segment in animals at the same time points after lesion. A sharp microelectrode was driven into the dorsal horn in $100 \mu \mathrm{m}$ steps from the superficial surface of the gray matter along three tracks, 400,600 , and $800 \mu \mathrm{m}$ lateral to the midline and field potentials recorded after $50 \mu \mathrm{A} \times 200 \mu$ s electrical stimulation of the intact $\mathrm{C} 7$ dorsal root both ipsilaterally and contralaterally. Figure 5 illustrates a quantitative threedimensional rendering of field potential recordings from intact (Fig. 5A,B), spared-root-lesioned and saline-treated (Fig. $5 D, E$ ), and spared-root-lesioned and ChABC-treated (Fig. 5G,H) animals recorded $14 \mathrm{~d}$ after lesion. The original data for these plots are shown in Figure 5, C, F, and $I$, respectively. Each plot represents the amplitude ( $y$-axis, and in color) and latency ( $z$-axis) of the activity recorded at certain depths in the dorsal horn ( $x$-axis). Intact sham-lesioned animals showed robust responses to C7 dorsal root stimulation (Fig. $5 A-C$ ); activity was recorded from the tip of the dorsal horn to $\sim 700$ $\mu \mathrm{m}$ into the gray matter; recording locations more ventral failed to show any rootstimulated responses. The average peak in amplitude of the $\mathrm{N}$-wave recorded from intact animals $(n=5)$ was approximately $-3 \mathrm{mV}$ (Fig. 5J). Contralateral to sparedroot injury, recorded activity was similar to that of intact animals after either saline (Fig. 5D) or ChABC (Fig. 5G) microinjections. However, ipsilateral to injury, saline-treated animals displayed greatly diminished activity (Fig. 5E). The average maximum amplitude of $\mathrm{N}$-wave recorded was approximately $-0.5 \mathrm{mV}$, significantly lower compared with intact animals at 7 , 14, and $28 \mathrm{~d}$ after lesion and treatment (Fig. $5 J)\left({ }^{\star} p<0.01\right.$, ANOVA). The latency of the $\mathrm{N}$-wave was significantly elevated in saline-treated animals compared with intact animals at all postlesion time points (Fig. $5 K)\left({ }^{*} p<0.05\right.$, ANOVA). Recordings made from animals that had received a microinjection of ChABC 

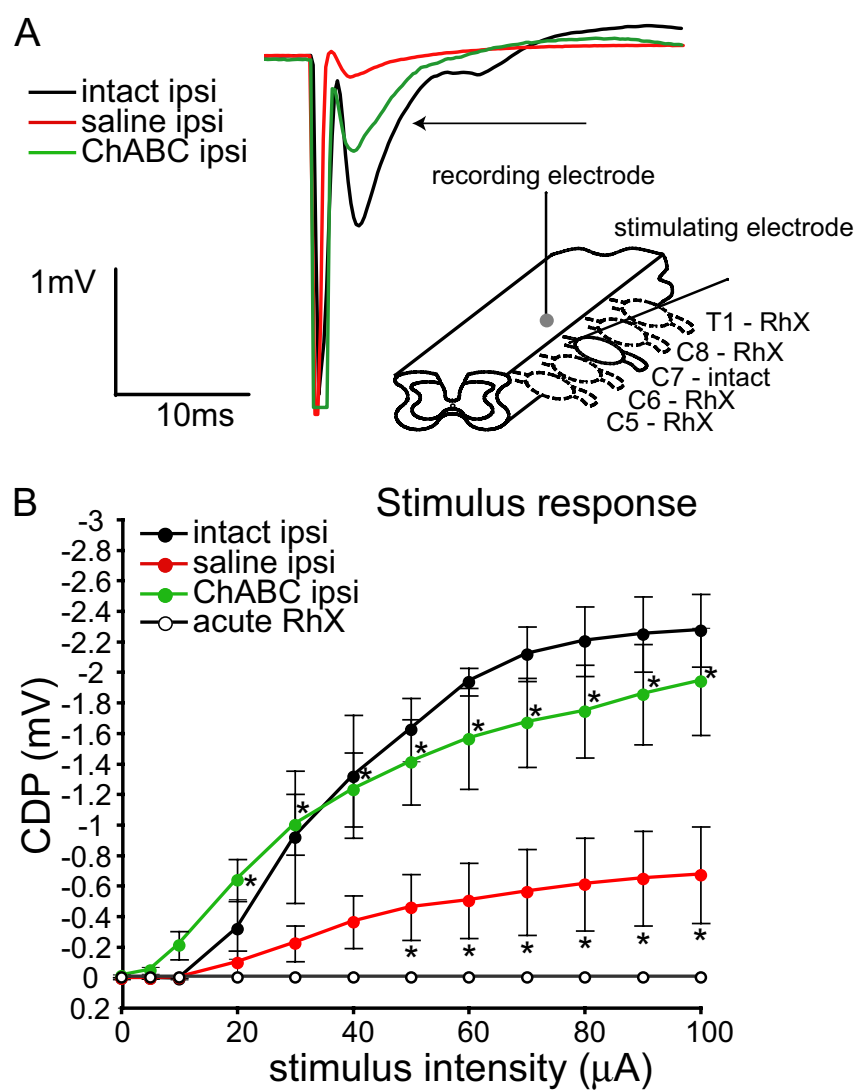

C

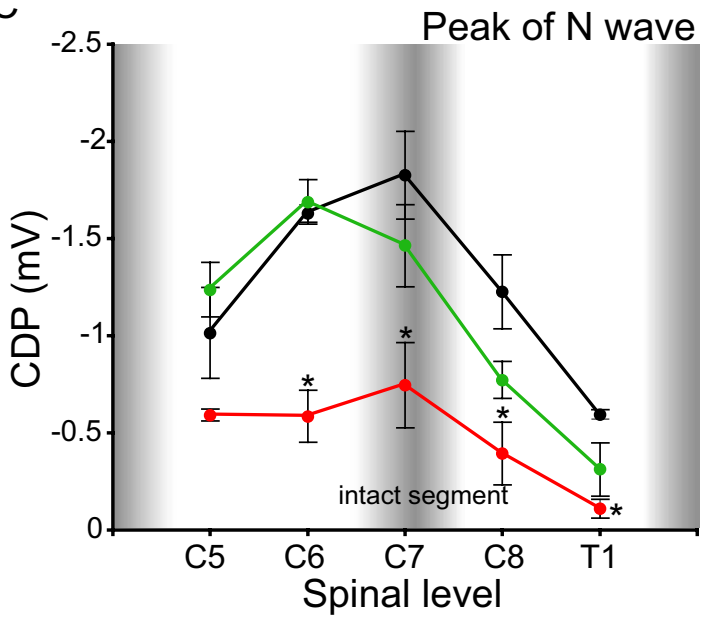

Figure 4. Restoration of postsynaptic activity after spared root lesion and chondroitinase ABC treatment. $\boldsymbol{A}$, Shown are examples of CDPs (arrow indicates $\mathrm{N}$-wave) recorded at the midline at $\mathrm{C} 7$ after electrical stimulation of the $\mathrm{C}$ dorsal root from sham-lesioned intact animals (black line) and spared-root-lesioned animals that received saline treatment (red line) or ChABC treatment (green line); schematic illustrates placement of stimulating and recording electrodes. $\boldsymbol{B}$, Stimulus-response curves were created by increasing the stimulus intensity in $10 \mu \mathrm{A}$ steps. A maximum response of $-2.0 \mathrm{mV}$ recorded in sham-lesioned animals plateaued at 60 $\mu \mathrm{A}$ (black circles); acute rhizotomy of the 77 dorsal root abolished activity (open circles). Spared-root lesion and saline treatment significantly reduced the maximum CDP recorded at $C 7$ (red circles), with a maximum recorded (DP of $-0.6 \mathrm{mV}$ ( ${ }^{*} p<0.05$, ANOVA). Spared-root lesion and ChABC-treated animals (green circles) exhibited CDPs significantly higher than saline-treated animals $\left({ }^{*} p<0.05\right)$, but were statistically indistinguishable from sham-lesioned animals. C, CDPs were recorded from adjacent spinal segments after spared-rootlesion; shading distinguishes intact segments from areas of deafferentation. Intact animals showed a peak in the N-wave of CDP at C7 (black circles); CDPs diminished when the recording electrode was moved either rostrally or caudally. Lesion- and saline-treated animals showed a significant decrease in CDP recorded at all spinal segments compared with sham-lesioned intact animals (red circles; ${ }^{*} p<0.05$, ANOVA). Lesion- and ChABC-treated animals displayed CDPs that were not significantly different from sham-lesioned controls ( $g r e e n$ circles). Error bars indicate SEM. concomitant to spared-root lesion exhibited a field potential profile broadly similar in magnitude and timing to intact animals (Fig. $5 \mathrm{H}$ ). The average maximum amplitude (Fig. 5J) of the $\mathrm{N}$-wave was significantly greater than saline-treated animals at 7,14 , and $28 \mathrm{~d}$ after lesion ( ${ }^{\#} p<0.01$, ANOVA), but not significantly different from contralateral records or intact animals at 7 and $14 \mathrm{~d}$ after lesion. The latency of the $\mathrm{N}$-wave from ChABC-treated animals was significantly reduced compared with saline-treated animals on days 7 and 14 after lesion ( ${ }^{\#} p \leq 0.05$, ANOVA), but indistinguishable from sham-lesioned animals at all time points (Fig. $5 K$ ). Thus, the restitution of evoked activity in ChABC-treated animals indicates a strengthening of synaptic connectivity within the $\mathrm{C} 7$ spinal segment after denervating injury.

\section{Single-unit recordings}

To confirm whether second-order neurons in the C7 spinal segment of ChABC-treated animals were receiving input from appropriate cutaneous fields, we made single-unit recordings in animals that had undergone spared-root lesion. We probed the intact C7 dorsal horn of intact animals and animals that had undergone spared-root surgery and either saline or ChABC treatment to identify receptive field properties of dorsal horn neurons to natural stimulation. We isolated 50 single units from intact sham-lesioned animals $(n=5)$ (summarized in Fig. 6A). Eighty-six percent of these units had discernable receptive fields, which were restricted to the medial paw and a strip of skin running along the lateral forelimb reaching the shoulder. Units were found both in the superficial and deep dorsal horn with equal frequency. Wide dynamic range units (Fig. 6E, G) were commonly found, which responded to brush, touch, and pinch stimulus (Fig. $6 E$ ). On electrical stimulation of the $\mathrm{C} 7$ dermatome, we were able to recruit units that produced PSTHs consistent with being driven by both A- and C-fibers (Fig. $6 G$ ).

Single-unit activity in animals that received spared-root injury and saline treatment is summarized in Figure $6 \mathrm{~A}$. In these animals, very few single units could be identified with clear receptive fields within the first 2 weeks after surgery. At these time points, units that were identified were mostly spontaneously active. By $21 \mathrm{~d}$ after lesion, it was possible to identify receptive fields in the majority of units. However, animals treated with ChABC after spared-root lesion showed considerably more evoked activity at earlier time points compared with saline-treated animals (Fig. 6A) $\left(p<0.005, \chi^{2}\right)$. We were able to identify 121 units from ChABC-treated animals (cf. 27 in the saline group), 31, 51, and 39 from 7,14 , and $28 \mathrm{~d}$ recovery groups ( $n=5$ /group), respectively. Units isolated from the $7 \mathrm{~d}$ recovery group were mostly spontaneously active $(90 \%)$ and $10 \%$ had clear receptive fields. However, $41 \%$ of units from $14 \mathrm{~d}$ recovery animals and $76 \%$ of $28 \mathrm{~d}$ recovery animals had receptive fields that were not significantly different from control animals. All receptive fields were centered on the $\mathrm{C} 7$ dermatome, but ChABC treatment was associated with the largest fields $\left({ }^{\star} p<0.05\right.$, ANOVA). Many of the units isolated from ChABC animals at 14 and $28 \mathrm{~d}$ after lesion displayed wide dynamic range characteristics (Fig. $6 F$ ). Transcutaneous electrical stimulation of the receptive fields typically induced responses in dorsal horn neurons consistent with activation by both $\mathrm{A}$ and $\mathrm{C}$ sensory fibers (Fig. $6 \mathrm{H}$ ). These data provide evidence that lesion-induced alterations in cutaneous processing were normalized after $\mathrm{ChABC}$ treatment. 

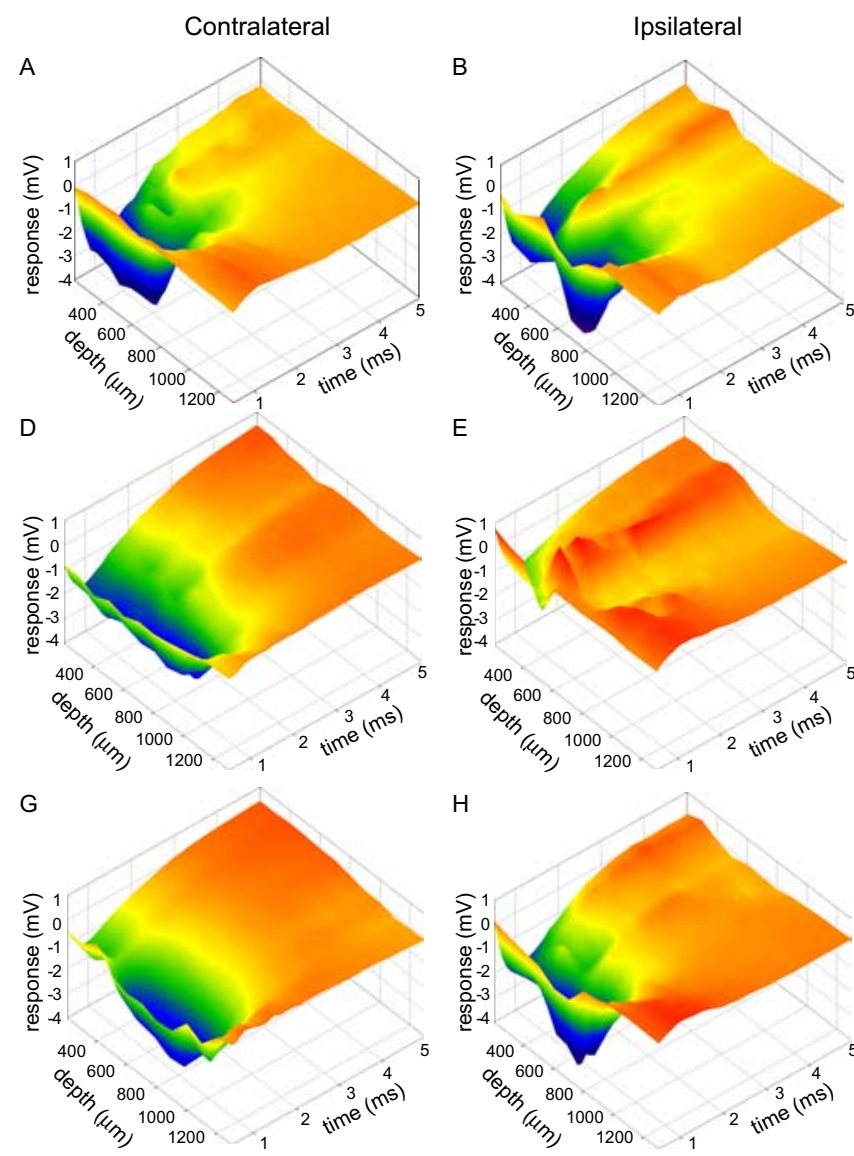

C

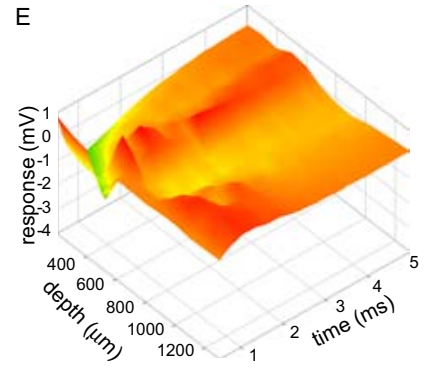

F
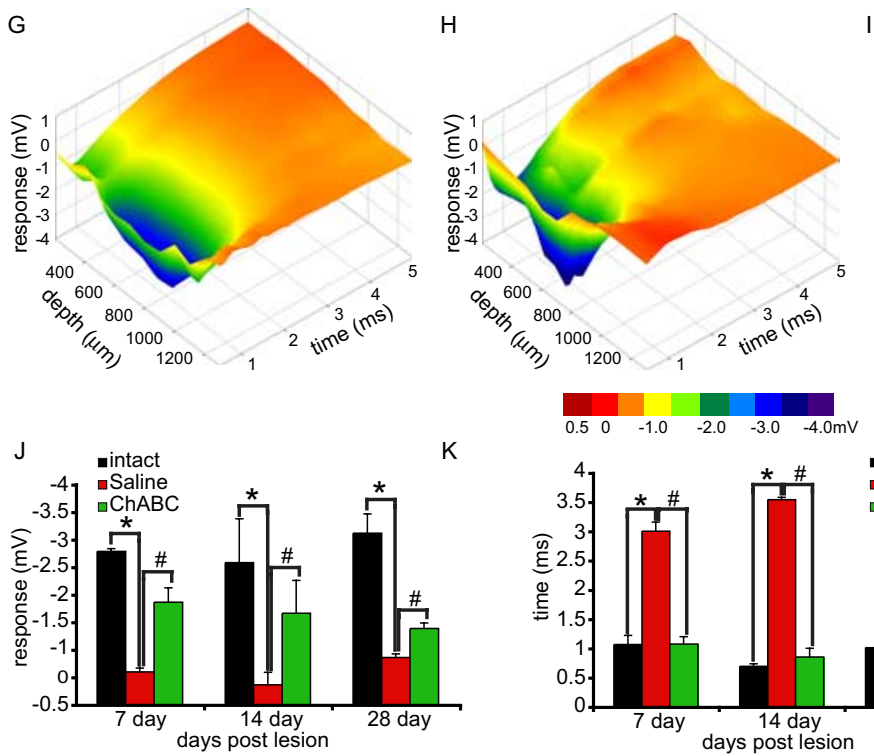

K

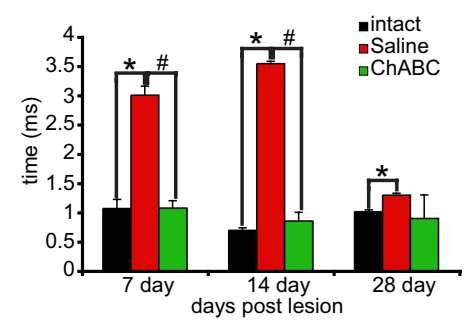

Figure 5. Restoration of spatially defined activity in the dorsal horn after spared-root lesion and chondroitinase $A B C$ treatment. $A-I$, Field potential recordings were made from 12 sites (surface of gray matter and descending in $100 \mu \mathrm{m}$ steps) along a track 400 $\mu \mathrm{m}$ lateral to the midline, 7, 14, and $28 \mathrm{~d}$ after sham, spared-root, and saline or ChABC treatment. Three-dimensional reconstructions of field potential recordings $(\boldsymbol{A}, \boldsymbol{B}, \boldsymbol{D}, \boldsymbol{E}, \boldsymbol{G}, \boldsymbol{H})$ illustrate activity changes (color coded; $0.5 \mathrm{mV}$, red, to $-4.0 \mathrm{mV}$, indigo) recorded at different depths ( $X$-axis) in spinal gray matter after electrical stimulation of the $C 7$ dorsal root (time, $z$-axis). Contralateral spinal cords from sham-lesioned $(\boldsymbol{A})$, lesion- and vehicle-treated $(\boldsymbol{D})$, and lesion- and $\mathrm{ChABC}$-treated $(\boldsymbol{G})$ animals illustrate equivalent activity profiles. The peak amplitude of the $\mathrm{N}$-wave and latency of $\mathrm{N}$-wave recorded from contralateral spinal cords were not significantly different between groups at any time point after lesion. Lesioned animals that received saline treatment illustrated significantly reduced activity ipsilateral to the lesion $(\boldsymbol{E}$; original trace shown in $\boldsymbol{F}, \boldsymbol{J})\left({ }^{*} p<0.05\right.$, ANOVA) compared with sham-lesioned animals ( $\boldsymbol{B}$; original trace, $\boldsymbol{C}$ ) at all time points after lesion $(\boldsymbol{J})$. The latency of the $\mathrm{N}$-wave was significantly elevated in saline-treated animals compared with sham-lesioned animals at all postlesion time points $(\boldsymbol{K})\left({ }^{*} p<0.05\right.$, ANOVA). In contrast, lesioned animals that received ChABC treatment illustrated robust activity in the dorsal horn ipsilateral to the lesion $(\boldsymbol{H}$; original trace, $\boldsymbol{I})$. Assessment of the peak amplitude of $\mathrm{N}$-wave showed that it was not significantly reduced compared with sham-lesioned animals at 7 and $14 \mathrm{~d}$ after lesion $(\boldsymbol{J})$ and was significantly higher than saline-treated animals at all postinjury time points ( $J$ ) ( ${ }^{\#} p<0.01$, ANOVA). The latency of the $\mathrm{N}$-wave from ChABC-treated animals was significantly reduced compared with saline-treated animals $(\boldsymbol{K})\left({ }^{\#} p \leq 0.05, \mathrm{ANOVA}\right)$, but not significantly different from sham-lesioned animals $(\boldsymbol{K})$. Error bars indicate SEM.

\section{Rhizotomy-induced reduction of mitogen-activated protein kinase phosphorylation is reversed by chondroitinase $\mathrm{ABC}$ treatment}

To ascertain whether dorsal horn neurons within deafferented spinal segments were receiving novel input from intact afferents, we delivered a noxious stimulus contralateral and ipsilateral to the lesion $14 \mathrm{~d}$ after treatment and assessed mitogen-activated protein (MAP) ki-

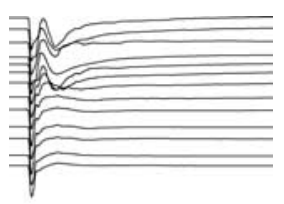

nase phosphorylation in second-order neurons. We injected capsaicin bilaterally into C7 dermatomes and counted the number of neurons that showed MAP kinase phosphorylation (i.e., pERK immunoreactivity). Capsaicin injection resulted in robust pERK immunoreactivity in second-order neurons in laminas I and II contralateral to the lesion in both saline- and ChABC-treated animals (Fig. $7 A, D, G$ ). However, lesioned animals that received saline showed a significant reduction $\left({ }^{*} p<0.001\right.$, ANOVA) in the number of pERK-IR neurons ipsilaterally at all spinal segments assessed (Fig. $7 B, C, G$ ). Lesioned animals that received ChABC illustrated significantly elevated numbers of pERK-IR neurons compared with salinetreated animals (Fig. $7 E-G)\left({ }^{*} p<0.01\right.$, ANOVA). The number of pERK-IR neurons ipsilateral to the lesion after ChABC treatment was not significantly different than the contralateral side at any spinal segment (Fig. 7G).

\section{Discussion}

The hypothesis that CSPGs within the CNS extracellular matrix (ECM) restrict adult spinal axon reorganization after SCI is supported by several major findings from this investigation. First, adult rats recovered forelimb sensory function after multiple cervical deafferentation (spared-root lesion) only after ChABC was infused into the dorsal horn. Second, intact primary afferent terminals sprout to maintain normal distribution after spared-root lesion in ChABC-treated animals only. Third, in vivo electrophysiological recording revealed that second-order neurons within the dorsal horn of deafferented spinal segments received novel input from intact C7 afferents after ChABC treatment. Fourth, noxious stimulation of the C7 dermatome in rats that had undergone sparedroot lesion caused MAP kinase phosphorylation in dorsal horn neurons within deafferented spinal segments after ChABC treatment. Therefore, we conclude that mitigating the inhibitory effects of intrinsic CSPGs via delivery of ChABC encourages reorganization of remaining spinal circuitry and subsequently restores function after injury.

\section{Degradation of intrinsic chondroitin sulfate proteoglycans}

Chondroitin sulfate proteoglycans along with hyaluronan and tenascins make up the ECM within the CNS (Bandtlow and Zimmermann, 2000). CSPGs are robustly distributed in both white and gray matter of the spinal cord (Tang et al., 2003). Whereas expression of CSPGs is elevated in the region of the DREZ after rhizotomy (Pindzola et al., 1993; Beggah et al., 2005), expression of neurocan and phos- 
phacan remains unchanged in the dorsal horn. By delivery of a bolus of ChABC into the dorsal horn, we were able to digest basally expressed CSPGs in spinal gray matter. This allowed us to test our central hypothesis that CSPGs exert a tonic inhibitory control over primary afferent terminal connectivity in the adult spinal cord. We rhizotomized dorsal roots $\mathrm{C} 5$, C6, C8, and $\mathrm{T} 1$, sparing $\mathrm{C} 7$. This paradigm creates a large area of deafferentation bisected by one set of intact terminals (C7). By using this spared-root preparation, we were able to isolate the function of the $\mathrm{C} 7$ sensory neurons innervating the forelimb. One potentially confounding issue with this surgical paradigm is the possibility that ChABC treatment would stimulate regeneration of dorsal root axons from rhizotomized levels, as two previous reports have shown (Steinmetz et al., 2005; Cafferty et al., 2007). We failed to observe any regeneration of rhizotomized axons into the spinal cord (supplemental Fig. 1, available at www. jneurosci.org as supplemental material). Our study does not contradict these two reports but rather highlights the possibility for spatially restricted efficacy of ChABC. Steinmetz et al. (2005) showed that a preconditioning inflammatory growth-enhancing stimulus was required before application of ChABC resulted in growth of rhizotomized axons into the spinal cord. Cafferty et al. (2007) transgenically expressed ChABC under the GFAP promoter; therefore, digestion of injury-induced CSPGs was restricted to the damaged DREZ. Both of these studies required delivery of $\mathrm{ChABC}$ to the damaged DREZ in order for axons to penetrate the spinal cord. In the current study, we delivered $\mathrm{ChABC}$ directly to spinal gray matter; negligible levels of ChABC-mediated diges-

tion were observed at the DREZs of injured segments. Therefore, any behavioral, histological, or electrophysiological change observed after ChABC treatment was likely mediated through alterations in the central connectivity of the remaining $\mathrm{C} 7$ afferent terminals.

\section{Restitution of sensory function}

The spared-root paradigm removes $>75 \%$ of sensory input from the rat forelimb, with remaining cutaneous input arising from C7 afferents only. Therefore, we assessed the ability of lesioned animals to perform spontaneous and trained somatosensory tasks, vertical exploration and tape removal. The spontaneous vertical exploration task highlights any asymmetry in use of forelimbs for upright postural weight support (Liu et al., 1999; Schallert et al., 2000). Rearing behavior is scored for each paw as the animal explores the walls of a transparent cylinder. This activity is similar to behavior in their home cages; therefore, repeated trials fail to bias paw usage (Schallert et al., 2000). Previous studies have shown that unilateral cortical aspiration (Woodlee et al., 2005), cervical hemisection (Schallert et al., 2000), and more subtle lesions of exclusive descending motor and ascending sensory spinal tracts (Liu et al., 1999, 2002; Webb and Muir, 2003) reduce ipsi- lateral paw usage (rats normally use both paws with equal frequency for initial contact with the cylinder wall and lateral movement). Consistent with these reports, we observed a shift in paw usage to the contralateral side in vehicle-treated animals after lesion. However, animals treated with $\mathrm{ChABC}$ recovered use of their ipsilateral forelimbs within $7 \mathrm{~d}$ of the lesion. Vehicle-treated animals showed improvements in paw usage asymmetry during the study but failed to reach presurgical levels; these improvements represent intrinsic plasticity of intact C7 terminals and/or plasticity within higher centers. The spatially restricted delivery of ChABC suggests that the additional recovery observed in these animals was a consequence of enhanced plasticity within local spinal circuitry. Furthermore, ChABC delivery reversed lesioninduced deficits observed in the sense component of the tape removal task. Animals that received vehicle treatment initially showed significantly elevated latencies in sensing the presence of the tape. However, latencies returned to baseline levels by the end of the testing period, again pointing to the potential for intrinsic plasticity to restore function. Interestingly, Cafferty et al. (2007) showed that adult wild-type mice that underwent cervical deafferentation from C5 to C8 (no spared root) recovered limited 

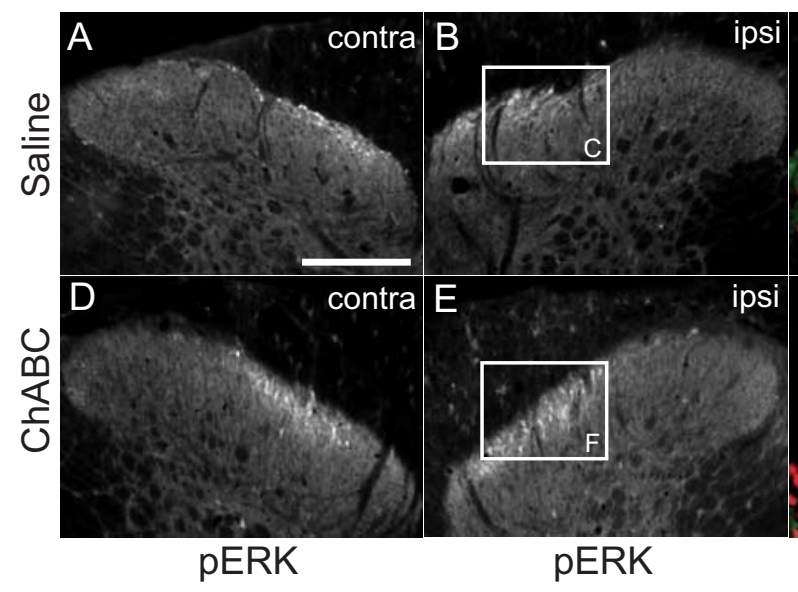

psi $\mathbf{C}$

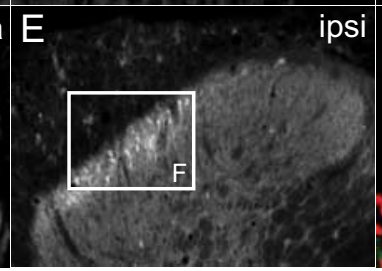

pERK

- Saline contra $\square$ ChABC contra

G

$\square$ Saline ipsi

$\square$ ChABC ipsi

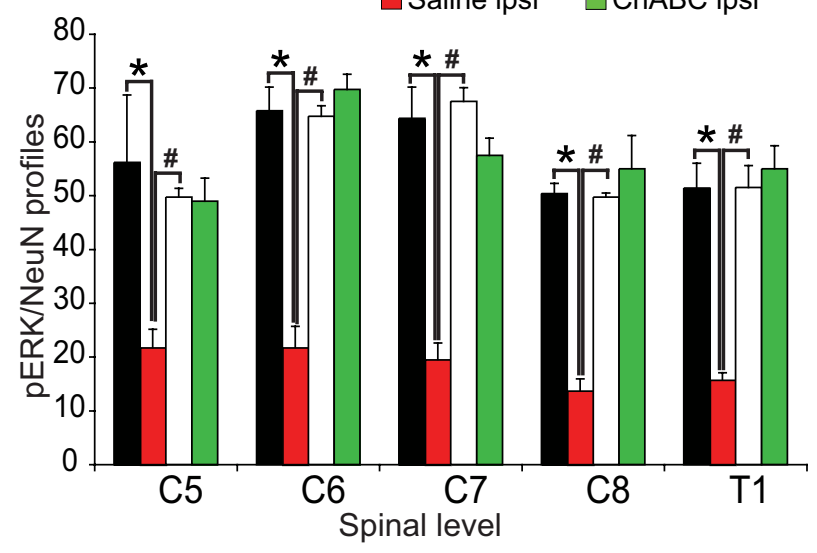

Figure 7. Peripheral noxious stimulus phosphorylates MAP kinase in deafferented spinal segments. $\boldsymbol{A}-\boldsymbol{F}$, Photomicrographs show capsaicin-induced phosphorylation of MAP kinase as detected by phosphospecific antibodies to ERK1/2 (pERK). PhosphoERK is detected in the medial aspect of lamina I and II contralateral to the spared-root lesion in saline $(\boldsymbol{A})$ - and $\mathrm{ChABC}(\boldsymbol{D})$-treated animals. Significantly reduced numbers of dual pERK-IR/NeuN + cells are seen ipsilateral after saline treatment (B, C). ChABCtreated animals maintain robust numbers of pERK-IR/NeuN + cells ipsilateral to the lesion $(\boldsymbol{E}, \boldsymbol{F}) . \boldsymbol{G}$, Quantification of the number and rostrocaudal distribution of dual pERK/NeuN-immunoreactive cells from $\mathrm{C} 5$ to $\mathrm{T} 1$ after bilateral noxious thermal stimulus. The number of $p E R K-I R$ cells on the contralateral side was the same between treatment groups at all segments. Lesion- and salinetreated animals illustrated a significant reduction $\left({ }^{*} p<0.005\right.$, ANOVA) in the number of $p E R K-I R$ cells on the ipsilateral side compared with the contralateral intact side at all segments. Lesion- and ChABC-treated animals showed a significant increase in the number of ipsilateral $p E R K-I R$ neurons compared with saline-treated animals ( ${ }^{*} p<0.005$, ANOVA). There was no significant difference in the number of $\mathrm{pERK} / \mathrm{NeuN}+$ cells between ipsilateral and contralateral sides after $\mathrm{ChABC}$ treatment at any spinal segment assessed. Error bars indicate SEM.

ability to sense the presence of adhesive tape, whereas animals that transgenically expressed ChABC at the DREZ recovered to prelesion levels. The slight improvement over time in wild-type mice was probably dependent on plasticity of remaining circuitry in the spinal cord. In the current study, the presence of one intact root is enough to restore function over time; the local delivery of $\mathrm{ChABC}$ accelerates this recovery, not by encouraging regeneration of damaged dorsal root fibers as in the study by Cafferty et al. (2007), but rather by reorganizing the connectivity of the intact C7 terminals. In this study, we observed a restoration in the terminal distribution of one subclass of C7 primary afferents after $\mathrm{ChABC}$ treatment. The functional significance of this anatomical reorganization can be more comprehensively assessed by sampling the effect their activation has on dorsal horn neurons. We chose to assess the molecular and electrophysiological consequences of $\mathrm{C} 7$ afferent activity after lesion and treatment on dorsal horn neurons, to develop an understanding of how ChABC was restoring sensory function.

\section{Restitution of central connectivity}

Peripheral noxious stimulation precipitates acute alteration in transcriptional and posttranslational events in secondorder neurons (Ji et al., 1999; Obata and Noguchi, 2004; Coggeshall, 2005). This molecular signature is particularly well studied in cutaneous nociceptive circuits (Harris, 1998). Animals that received vehicle infusion after spared-root lesion illustrated a significant reduction in MAP kinase activation in dorsal horn neurons of $\mathrm{C} 7$ and deafferented segments. Animals treated with ChABC illustrated MAP kinase activation at all spinal levels in a manner indistinguishable from their contralateral wholly intact side. These data point to ChABC-dependent formation of novel or enhancement of existing connections between C7 afferents and dorsal horn neurons located in adjacent spinal segments.

To correlate $\mathrm{ChABC}$-mediated reorganization of $\mathrm{C} 7$ connectivity and restitution of sensory function, we completed extensive in vivo electrophysiological assessment of the dorsal horn of intact and deafferented spinal segments. Cord dorsum potentials recorded from lesioned animals treated with vehicle after electrical C7 root stimulation illustrated a reduction in activity within both deafferented and intact spinal segments. A more comprehensive investigation of the intact segment confirmed a reduction of $\mathrm{C} 7$ afferent stimulated activity throughout the dorsal horn, which was apparent up to $28 \mathrm{~d}$ after lesion. This wholesale reduction in activity even within the intact segment is consistent with the fact that there is extensive overlap of primary afferent terminals from adjacent dorsal roots in the cervical cord (Wilson and Kitchener, 1996). Spared-root lesions prepared in the lumbar spinal cord of adult cats showed reduced activity acutely after deafferentation (Basbaum and Wall,

1976; Brinkhus and Zimmermann, 1983). Furthermore, electron-microscopic investigation of synaptic terminals within lamina II of the intact L6 spinal segment in cats that had undergone extensive deafferentation from L1 to S2 showed a reduction in complex terminal density (Zhang et al., 1995). Therefore, dorsal horn neurons in both the deafferented and intact segments lose synaptic input and are consequently hypoactive, at least for some time. Strikingly, lesioned animals that received $\mathrm{ChABC}$ failed to show a decrease in activity in deafferented or intact segments. Mass wave recording throughout the dorsal horn appeared indistinguishable of intact controls.

Restitution of "normal" activity within the spinal cord of ChABC-treated animals is consistent with our behavioral data. However, single-unit recordings from lesioned animals revealed that $\mathrm{ChABC}$-mediated synaptic reorganization was probably not a reconstitution of normal activity but rather the emergence of substitute function.

Single units recorded from lesioned animals treated with ve- 
hicle were mostly spontaneously active acutely after injury, with the majority beginning to demonstrate some peripheral drive from diffuse receptive fields at $28 \mathrm{~d}$ after lesion. At this time point, the average receptive field size of these units was significantly lower than intact animals because of reduced efficacy after lesion of adjacent roots. Furthermore, many receptive fields of C7 spinal neurons will normally be constructed from several dermatomes and these will be reduced as a consequence of the lesion. ChABC-treated animals, however, had an average receptive field size that was larger than intact controls. This observation may appear to suggest peripheral anatomical reorganization. However, receptive field size is not determined strictly by anatomy. Receptive field size can expand in most dorsal horn neurons by reducing inhibition or increasing excitation, that is, most neurons have a subliminal fringe to their receptive field that can be unmasked by a variety of maneuvers. For example, repetitively stimulating the receptive field of many neurons will lead to progressive increases in size over seconds and not requiring anatomical remodeling. Subliminal inputs that are intact are quite plausibly recruited after $\mathrm{ChABC}$ treatment.

\section{Mechanisms of recovery}

A combination of two mechanisms can explain these observations (summarized in Fig. 8). First, ChABC has unmasked activity in silent synapses in both intact and deafferented spinal segments. The existence of nonfunctional connections has been extensively studied by Wall and colleagues over a long period and may reflect remnants of pioneering axons in the developmental phase that have not been fully withdrawn (Wall, 1995). Therefore synapses that were previously weak or ineffective may become stronger because of retraction of competing, now degenerating terminals. Furthermore, a role for silent connections has been proposed under chronic pathological conditions in which nociceptive afferents may become responsive to noxious stimuli (McMahon and Koltzenburg, 1990). Second, intraspinal sprouting of intact primary afferents may make novel connections on dorsal horn neurons that have lost synaptic input. Although collateral sprouting has been reported in both the cat and rat, its existence remains controversial (Liu and Chambers, 1958; Murray and Goldberger, 1974; Goldberger and Murray, 1982). Our observations may reflect either unmasking and/or sprouting.

There is now abundant evidence to support the hypothesis that injury upregulated CSPGs arrest axonal regeneration after SCI (Busch and Silver, 2007) and that digestion of CSPGs with ChABC either alone or in combination results in significant functional recovery (Yiu and He, 2006). However, the role of basally expressed CSPGs in the spinal cord is less clear. Recent elegant studies have shown that $\mathrm{ChABC}$ can encourage sprouting of intact spinal axons into denervated territories (Barritt et al., 2006; Massey et al., 2006, 2008). However, the function of these nascent pathways remains to be fully characterized. We have now demonstrated that basally expressed CSPGs in the adult spinal cord inhibit the reorganization of intact spinal circuits, which after digestion with $\mathrm{ChABC}$ can enhance recovery. Anatomical evidence of axonal growth after rodent SCI is modest compared with the observed functional recovery (Bradbury and McMahon, 2006). Most investigators ascribe this discrepancy to reorganization of intact circuitry either in the brain or the spinal cord (Bradbury and McMahon, 2006; Maier and Schwab, 2006). This study is the first to provide functional evidence to support a meaningful role for reorganization of intact spinal systems after SCI and provides a clear rationale for targeting these circuits to develop additional strategies to enhance functional restoration in patients with brain and spinal cord injury.

\section{References}

Asher RA, Morgenstern DA, Fidler PS, Adcock KH, Oohira A, Braistead JE, Levine JM, Margolis RU, Rogers JH, Fawcett JW (2000) Neurocan is upregulated in injured brain and in cytokine-treated astrocytes. J Neurosci 20:2427-2438.

Asher RA, Morgenstern DA, Shearer MC, Adcock KH, Pesheva P, Fawcett JW (2002) Versican is upregulated in CNS injury and is a product of oligodendrocyte lineage cells. J Neurosci 22:2225-2236.

Bandtlow CE, Zimmermann DR (2000) Proteoglycans in the developing brain: new conceptual insights for old proteins. Physiol Rev 80:1267-1290. 
Baptiste DC, Fehlings MG (2007) Update on the treatment of spinal cord injury. Prog Brain Res 161:217-233.

Bareyre FM, Kerschensteiner M, Raineteau O, Mettenleiter TC, Weinmann O, Schwab ME (2004) The injured spinal cord spontaneously forms a new intraspinal circuit in adult rats. Nat Neurosci 7:269-277.

Barritt AW, Davies M, Marchand F, Hartley R, Grist J, Yip P, McMahon SB, Bradbury EJ (2006) Chondroitinase ABC promotes sprouting of intact and injured spinal systems after spinal cord injury. J Neurosci 26:10856-10867.

Basbaum AI, Wall PD (1976) Chronic changes in the response of cells in adult cat dorsal horn following partial deafferentation: the appearance of responding cells in a previously non-responsive region. Brain Res 116:181-204.

Beggah AT, Dours-Zimmermann MT, Barras FM, Brosius A, Zimmermann DR, Zurn AD (2005) Lesion-induced differential expression and cell association of Neurocan, Brevican, Versican V1 and V2 in the mouse dorsal root entry zone. Neuroscience 133:749-762.

Berardi N, Pizzorusso T, Maffei L (2004) Extracellular matrix and visual cortical plasticity: freeing the synapse. Neuron 44:905-908.

Bradbury EJ, McMahon SB (2006) Spinal cord repair strategies: why do they work? Nat Rev Neurosci 7:644-653.

Bradbury EJ, Moon LD, Popat RJ, King VR, Bennett GS, Patel PN, Fawcett JW, McMahon SB (2002) Chondroitinase ABC promotes functional recovery after spinal cord injury. Nature 416:636-640.

Brinkhus HB, Zimmermann M (1983) Characteristics of spinal dorsal horn neurons after partial chronic deafferentation by dorsal root transection. Pain 15:221-236.

Busch SA, Silver J (2007) The role of extracellular matrix in CNS regeneration. Curr Opin Neurobiol 17:120-127.

Cafferty WB, Strittmatter SM (2006) The Nogo-Nogo receptor pathway limits a spectrum of adult CNS axonal growth. J Neurosci 26:12242-12250.

Cafferty WB, Yang SH, Duffy PJ, Li S, Strittmatter SM (2007) Functional axonal regeneration through astrocytic scar genetically modified to digest chondroitin sulfate proteoglycans. J Neurosci 27:2176-2185.

Caggiano AO, Zimber MP, Ganguly A, Blight AR, Gruskin EA (2005) Chondroitinase ABCI improves locomotion and bladder function following contusion injury of the rat spinal cord. J Neurotrauma 22:226-239.

Coggeshall RE (2005) Fos, nociception and the dorsal horn. Prog Neurobiol 77:299-352.

Davies SJ, Goucher DR, Doller C, Silver J (1999) Robust regeneration of adult sensory axons in degenerating white matter of the adult rat spinal cord. J Neurosci 19:5810-5822.

Fox K, Caterson B (2002) Neuroscience. Freeing the bain from the perineuronal net. Science 298:1187-1189.

Goldberger ME, Murray M (1982) Lack of sprouting and its presence after lesions of the cat spinal cord. Brain Res 241:227-239.

Harris JA (1998) Using c-fos as a neural marker of pain. Brain Res Bull 45:1-8.

Hensch TK (2004) Critical period regulation. Annu Rev Neurosci 27:549-579.

Ji RR, Baba H, Brenner GJ, Woolf CJ (1999) Nociceptive-specific activation of ERK in spinal neurons contributes to pain hypersensitivity. Nat Neurosci 2:1114-1119.

Jones LL, Yamaguchi Y, Stallcup WB, Tuszynski MH (2002) NG2 is a major chondroitin sulfate proteoglycan produced after spinal cord injury and is expressed by macrophages and oligodendrocyte progenitors. J Neurosci 22:2792-2803.

Jones LL, Margolis RU, Tuszynski MH (2003) The chondroitin sulfate proteoglycans neurocan, brevican, phosphacan, and versican are differentially regulated following spinal cord injury. Exp Neurol 182:399-411.

Kim JE, Liu BP, Park JH, Strittmatter SM (2004) Nogo-66 receptor prevents raphespinal and rubrospinal axon regeneration and limits functional recovery from spinal cord injury. Neuron 44:439-451.

Liu BP, Cafferty WB, Budel SO, Strittmatter SM (2006) Extracellular regulators of axonal growth in the adult central nervous system. Philos Trans R Soc Lond B Biol Sci 361:1593-1610.

Liu CN, Chambers WW (1958) Intraspinal sprouting of dorsal root axons; development of new collaterals and preterminals following partial denervation of the spinal cord in the cat. AMA Arch Neurol Psychiatry 79:46-61.

Liu Y, Kim D, Himes BT, Chow SY, Schallert T, Murray M, Tessler A, Fischer I (1999) Transplants of fibroblasts genetically modified to express BDNF promote regeneration of adult rat rubrospinal axons and recovery of forelimb function. J Neurosci 19:4370-4387.

Liu Y, Himes BT, Murray M, Tessler A, Fischer I (2002) Grafts of BDNF- producing fibroblasts rescue axotomized rubrospinal neurons and prevent their atrophy. Exp Neurol 178:150-164.

Maier IC, Schwab ME (2006) Sprouting, regeneration and circuit formation in the injured spinal cord: factors and activity. Philos Trans R Soc Lond B Biol Sci 361:1611-1634.

Massey JM, Hubscher CH, Wagoner MR, Decker JA, Amps J, Silver J, Onifer SM (2006) Chondroitinase ABC digestion of the perineuronal net promotes functional collateral sprouting in the cuneate nucleus after cervical spinal cord injury. J Neurosci 26:4406-4414.

Massey JM, Amps J, Viapiano MS, Matthews RT, Wagoner MR, Whitaker CM, Alilain W, Yonkof AL, Khalyfa A, Cooper NG, Silver J, Onifer SM (2008) Increased chondroitin sulfate proteoglycan expression in denervated brainstem targets following spinal cord injury creates a barrier to axonal regeneration overcome by chondroitinase ABC and neurotrophin-3. Exp Neurol 209:426-445.

McGee AW, Yang Y, Fischer QS, Daw NW, Strittmatter SM (2005) Experience-driven plasticity of visual cortex limited by myelin and Nogo receptor. Science 309:2222-2226.

McMahon SB, Koltzenburg M (1990) Novel classes of nociceptors: beyond Sherrington. Trends Neurosci 13:199-201.

Moon LD, Asher RA, Rhodes KE, Fawcett JW (2001) Regeneration of CNS axons back to their target following treatment of adult rat brain with chondroitinase ABC. Nat Neurosci 4:465-466.

Murray M, Goldberger ME (1974) Restitution of function and collateral sprouting in the cat spinal cord: the partially hemisected animal. J Comp Neurol 158:19-36.

Obata K, Noguchi K (2004) MAPK activation in nociceptive neurons and pain hypersensitivity. Life Sci 74:2643-2653.

Pindzola RR, Doller C, Silver J (1993) Putative inhibitory extracellular matrix molecules at the dorsal root entry zone of the spinal cord during development and after root and sciatic nerve lesions. Dev Biol 156:34-48.

Pizzorusso T, Medini P, Berardi N, Chierzi S, Fawcett JW, Maffei L (2002) Reactivation of ocular dominance plasticity in the adult visual cortex. Science 298:1248-1251.

Pizzorusso T, Medini P, Landi S, Baldini S, Berardi N, Maffei L (2006) Structural and functional recovery from early monocular deprivation in adult rats. Proc Natl Acad Sci U S A 103:8517-8522.

Schallert T, Fleming SM, Leasure JL, Tillerson JL, Bland ST (2000) CNS plasticity and assessment of forelimb sensorimotor outcome in unilateral rat models of stroke, cortical ablation, parkinsonism and spinal cord injury. Neuropharmacology 39:777-787.

Steinmetz MP, Horn KP, Tom VJ, Miller JH, Busch SA, Nair D, Silver DJ, Silver J (2005) Chronic enhancement of the intrinsic growth capacity of sensory neurons combined with the degradation of inhibitory proteoglycans allows functional regeneration of sensory axons through the dorsal root entry zone in the mammalian spinal cord. J Neurosci 25:8066-8076.

Tang X, Davies JE, Davies SJ (2003) Changes in distribution, cell associations, and protein expression levels of NG2, neurocan, phosphacan, brevican, versican V2, and tenascin-C during acute to chronic maturation of spinal cord scar tissue. J Neurosci Res 71:427-444.

Tessier-Lavigne M (2002) Wiring the brain: the logic and molecular mechanisms of axon guidance and regeneration. Harvey Lect 98:103-143.

Wall PD (1995) Do nerve impulses penetrate terminal arborizations? A prepresynaptic control mechanism. Trends Neurosci 18:99-103.

Webb AA, Muir GD (2003) Unilateral dorsal column and rubrospinal tract injuries affect overground locomotion in the unrestrained rat. Eur J Neurosci 18:412-422.

Wilson P, Kitchener PD (1996) Plasticity of cutaneous primary afferent projections to the spinal dorsal horn. Prog Neurobiol 48:105-129.

Woodlee MT, Asseo-García AM, Zhao X, Liu SJ, Jones TA, Schallert T (2005) Testing forelimb placing "across the midline" reveals distinct, lesiondependent patterns of recovery in rats. Exp Neurol 191:310-317.

Yick LW, Wu W, So KF, Yip HK, Shum DK (2000) Chondroitinase ABC promotes axonal regeneration of Clarke's neurons after spinal cord injury. Neuroreport 11:1063-1067.

Yiu G, He Z (2006) Glial inhibition of CNS axon regeneration. Nat Rev Neurosci 7:617-627.

Zhang B, Goldberger ME, Wu LF, Murray M (1995) Plasticity of complex terminals in lamina II in partially deafferented spinal cord: the cat spared root preparation. Exp Neurol 132:186-193.

Zuo J, Neubauer D, Dyess K, Ferguson TA, Muir D (1998) Degradation of chondroitin sulfate proteoglycan enhances the neurite-promoting potential of spinal cord tissue. Exp Neurol 154:654-662. 